\title{
INVESTIGATING THE SIGNATURES OF LONG-RANGE PERSISTENCE IN SEISMIC SEQUENCES ALONG CIRCUM-PACIFIC SUBDUCTION ZONES
}

\author{
Daniel Brito de Freitas ${ }^{1}$, George Sand L. A. de França², Thais M. Scherrer ${ }^{3}$, \\ Carlos S. Vilar ${ }^{4}$ and Raimundo Silva ${ }^{5}$
}

\begin{abstract}
In the present paper, we analyze the signatures of long-range persistence in seismic sequences along Circum-Pacific subduction zones, from Chile to Kermadec, extracted from the National Earthquake Information Center (NEIC) catalog. This region, known as the Pacific Ring of Fire, is the world's most active fault line, containing about $90 \%$ of the world's earthquakes. We used the classical rescaled range $(R / S)$ analysis to estimate the long-term persistence signals derived from a scaling parameter called the Hurst exponent, $H$. We measured the referred exponent and obtained values of $H>0.5$, indicating that a long-term memory effect exists. We found a possible fractal relationship between $H$ and the $b_{s}(q)$-index, which emerges from the nonextensive Gutenberg-Richter law as a function of the asperity. Therefore, $H$ can be associated with a mechanism that controls the level of seismic activity. Finally, we concluded that the dynamics associated with fragment-asperity interactions can be classified as a self-affine fractal phenomenon.
\end{abstract}

Keywords: applied geophysics, fault and fracture analysis, mathematics applied to geophysics, seismology geostatistics.

RESUMO. No presente artigo, analisamos as assinaturas de persistência long-range nas sequências sísmicas ao longo das zonas de subducção Circum-Pacific, do Chile até Kermadec, extraídas do catálogo do National Earthquakes Information Center (NEIC). Esta região, conhecida como Anel de Fogo do Pacífico, é a linha de falhas mais ativa do mundo, contendo cerca de $90 \%$ dos terremotos do mundo. Usamos a análise clássica $R / S$ para estimar a assinatura de persistência a longo prazo derivada do parâmetro de escalonamento chamado expoente de Hurst, $H$. Como principal objeto de estudo, medimos o referido expoente e obtivemos todos os valores de $H>0,5$, indicando que existe um efeito de memória de longo prazo. Encontramos uma possível relação entre $H$ e 0 índice $b_{s}(q)$ que emerge da lei de Gutenberg-Richter não-extensiva como uma função da aspereza, isto é, $H$ pode estar associado ao mecanismo que controla o nível de atividade dos terremotos. Finalmente, concluímos que a dinâmica associada às interações fragilidade-aspereza pode ser classificada como um fenômeno fractal auto-afim.

Palavras-chave: geofísica aplicada, análise de falhas e fraturas, matemática aplicada à geofísica, sismologia geoestatística.

\footnotetext{
Corresponding author: Daniel Brito de Freitas

${ }^{1}$ Universidade Federal do Ceará, Departamento de Física Caixa Postal 6030, Campus do Pici, 60455-900 Fortaleza, CE, Brazil - E-mail: danielbrito@fisica.ufc.br

2 Observatório Sismológico-IG/UnB, Campus Universitário Darcy Ribeiro SG 13 Asa Norte, 70910-900 Brasília, DF, Brazil - E-mail: georgesand@unb.br

${ }^{3}$ Conselho Nacional de Desenvolvimento Científico e Tecnológico, CNPq, Brazil - E-mail: t_scherrer@yahoo.com.br

${ }^{4}$ Universidade Federal da Bahia, Instituto de Física Campus Universitário de Ondina, 40210-340 Salvador, BA, Brazil - E-mail: vilar@ufba.br

5 Universidade Federal do Rio Grande do Norte, Departamento de Física, 59072-970, Natal, RN, Brazil - E-mail: raimundo@fisica.ufrn.br
} 


\section{INTRODUCTION}

Geophysical signals often fluctuate in an irregular and complex way over time, and present inhomogeneous variations and extreme events, such as irregular rupture propagation and non-uniform distributions of rupture velocity, stress drop, and coseismic slip (Telesca et al., 2005). The presence of scaling properties in geophysical data points out that methods of fractal analysis based on the long-term correlations may provide a viable way to investigate the pattern of magnitudes in an episode of seismicity (Li \& Chen, 2001). The Earth's tectonic activity is due to very complex mechanisms that involve many variables, such as deformation, rupture, released energy, land features, and heterogeneity on the seismogenic plate interface (Kawamura et al., 2012; Scherrer et al., 2015). To analyze such a complex time series, there is a plethora of different methods and tools that can be used to better describe the dynamical properties of earthquakes (Omori, 1894; Gutenberg \& Richter, 1944).

Several statistical methods are reported in the scientific literature, which use the concept of fractality. Among them, we can find methods based on self-similar and self-affine fractals, such as the box dimension (Peitgen et al., 2004), the detrended fluctuation analysis (DFA) (Peng et al., 1992), the detrending moving average analysis (DMA) (Alessio et al., 2002), and the scaled windowed variance analysis (SWVA) (Mandelbrot, 1985).

In the present paper, we will characterize the dynamics of earthquakes by calculating the Hurst exponent, where features such as long-range persistence can be investigated (Seuront, 2009). Time series are quantified by their persistence or anti-persistence signature. Generally speaking, persistence can be described in terms of range, short- and long-range, where the memory can be classified as weak and strong, respectively. Statistical analysis is characterized by power-law distributions and can be a powerful tool for examining the temporal fluctuations at different scales when applied to earthquake magnitude time series Telesca (2016).

In the present paper, we investigate the signatures of long-range persistence that are present in the earthquake magnitude times series for the Circum-Pacific subduction zones, and have been already been processed by Scherrer et al. (2015). Our study applied the rescaled range $(R / S)$ analysis as a self-affine fractal method to the magnitude time series (de Freitas et al., 2013). The characteristic measure of the $R / S$ analysis is the Hurst exponent, denoted by $H$. It is worth noting the universal character of the $R / S$ method in the analysis of the behavior of fluctuations. Many studies in different subject areas (e.g., economy, neuroscience, and astrophysics) have shown that the so-called Hurst exponent extracted from the $R / S$ analysis provides a robust and powerful statistical method to characterize nonstationary fluctuations at different timescales (Li \& Chen, 2001; Suyal et al., 2009; de Freitas et al., 2013). More recently, de Freitas et al. (2013) found a Hurst exponent of 0.87 for the San Andreas fault, which indicated a strong long-term persistence. Other studies (e.g., Li \& Chen, 2001) also point out that the Hurst exponent is greater than 0.5 , indicating a persistent behavior.

Our main interest is to investigate a possible correlation between scaling properties (controlled by interaction asperities) (Lay \& Kanamori, 2013) and the Hurst exponent estimated from the $(R / S)$ analysis (Hurst, 1951; Mandelbrot \& Wallis, 1969b). In this paper, we show a detailed investigation of the rescaled range analysis to search for long-range correlations using the Hurst exponent in subduction zones along the Pacific Ring of Fire, the most seismically active region on Earth Scherrer et al. (2015). In particular, each zone was described using a general classification defined by an asperity model described by (Scherrer et al., 2015). Our main aim is to investigate two questions. Firstly, is there a correlation between $H$-value and the Circum-Pacific subduction zones? Secondly, is there any connection between the fractal parameter and the asperity model from Lay \& Kanamori (2013)?

Our paper is organized as follows, in the next Section, we describe the Hurst method used in our study and a brief discussion about the nonextensive formalism. In Catalog Data, we present a sample of our seismic catalog. The main results and their physical implications are presented in Results and Discussions. Finally, closing remarks are drawn in Conclusions.

\section{STATISTICAL BACKGROUND}

\section{Hurst effect}

As pointed out by Seuront (2009), there are various methods to describe the behavior of a time series using self-affine fractals. Mandelbrot \& Wallis (1969a,b) introduced the concept of fractional Brownian motion (fBm) as a generalization of Brownian motion, which assumes the motion of an object is a union of rescaled copies of itself uniformly distributed in all directions, i.e., a self-similar fractal. In contrast, fBm considers the rescaling of the copies of itself is dependent on the direction, denoted as a self-affine (Seuront, 2009). As mentioned by Seuront (2009), a series of successive increments in a $\mathrm{fBm}$ defines a fractional Gaussian noise (fGn). This other type of time series based on the increment of a fBm yields a stationary signal with a mean of zero. 
Strictly speaking, a geophysical data set can be modeled as either a fGn-like or a noise-like time series (de Freitas et al., 2013).

The scientific literature points out different techniques for exploring time series using a fractal approach. In general, the characteristics of a time series include a wider spectrum of complex measurements due to nonstationarity, nonlinearity, fractality, stochasticity, periodicity, chaos, and so on Tang et al. (2015). A powerful fractal technique for dealing with these assumptions is the Hurst analysis. As proposed by Hurst (1951), we will focus on rescaled range analysis, also known as $R / S$ analysis.

\section{Fractal index measured using the $R / S$ method}

As described by Hurst (1951), a fractal analysis is used to measure the long-term memory or correlation of a time series. The method developed by Hurst (1951), known as the rescaled range analysis (hereafter $R / S$ analysis), is the method adopted here to estimate the Hurst exponent $H$. Our aim is to verify the capability of the $R / S$ method to distinguish the different properties in the subduction zones through the behavior of $H$ and its possible correlation with the constant $b_{s}$, extracted from the generalized Gutenberg-Richter law, as measured by Sarlis et al. (2010). The aforementioned constant is the slope of the cumulative distribution number of earthquakes with magnitude greater than $m$ (for further details, see Eq. 9 from Sarlis et al., 2010).

Firstly, we consider a time series given by $x(t):=$ $x(1), x(2), \ldots, x(N)$ with a time-window of length $N$. Following the same procedure mentioned by (de Freitas et al., 2013), the analysis starts with two elements, $n=2$, and for each iteration one element is added until $n=N$ (i.e., the whole time series). In each cumulative window, we can measure two quantities that are denoted by $R(n)$ (the distance between the minimum and the maximum value of the accumulated deviations from the mean of $x(t)$ within the window of length $n$ ) and $S(n)$ (the standard deviation of the values of $x(t)$ in this same time-window). From a mathematical point-view, $R(n)$ can be written as:

$$
R(n)=\max _{1 \leq t \leq n} X(t, n)-\min _{1 \leq t \leq n} X(t, n),
$$

where the variable $X(t, n)$ is defined as $X(t, n)=$ $\sum_{t=1}^{n}\left(x(t)-\langle x(t)\rangle_{n}\right)$. Therefore, the long-trend is removed using the mean over the values of $x(t)$ within the window. $S(n)$ is defined as:

$$
S(n)=\left[\frac{1}{n} \sum_{t=1}^{n}\left(x(t)-\langle x(t)\rangle_{n}\right)^{2}\right]^{1 / 2} .
$$

Based on these two parameters, we define the $R / S$ statistics of the fluctuations in the time series as a power-law dependence over a box of $n$ elements given by:

$$
\frac{R(n)}{S(n)}=k n^{H},
$$

where $k$ is a constant and the Hurst index $(H)$ can be measured by fitting the slope of the $\log -\log$ plot of $R(n) / S(n)$ versus $n$ :

$$
\log \left[\frac{R(n)}{S(n)}\right] \sim H \log (n) .
$$

A time series described by the $R / S$ statistic is said to be fractal if the $R / S$ curve is a perfectly straight line; this means that the residual between this straight line and the $R / S$ curve must be null. If the $R / S$ curve presents some fluctuations (not large however) around the straight line that estimates $H$, this could be because the data are not ideal but observational and so are affected by measurement errors that make the curve $R / S$ depart (but not with large amplitude) from a straight line. If the $R / S$ curve does not follow a straight line or if the departures from the straight line are very large the object is not fractal.

The Hurst exponent quantifies the probability that a given event in a process is followed by a similar event. As mentioned by de Freitas et al. (2013), the $R / S$ method is used to calculate the scaling exponent, $H$, to give a quantitative measure of the persistence of a signal. Their typical values are: $0.5<H<1$ which indicates a persistence or long memory process, $H=0.5$ which indicates an uncorrelated process, and $0<H<0.5$ denotes anti-correlation. In a geophysical scenario, earthquakes bear dual features of randomicity and regularity, and therefore a $H$-value of between 0.5 and 1 is expected (de Freitas et al., 2013).

\section{Nonextensive framework}

Inspired by multifractals ${ }^{1}$, Tsallis (1988) proposed a new concept of entropy extracted from a generalization of the Boltzmann-Gibbs (BG) entropy. This generalized entropy is defined as $S_{q}$ and is given by the following equation:

$$
S_{q}=-k_{B} \frac{1-\sum_{i=1}^{W} p_{i}^{q}}{q-1}
$$

\footnotetext{
${ }_{1}^{1}$ Multifractals are a generalization of fractal systems in which only a single exponent (e.g., for instance, the Hurst exponent) is not enough to describe their dynamics. Further details can be found in Mandelbrot \& Wallis (1969a,b); de Freitas et al. (2017).
} 
which is based on the entropic index $q$ which measures the degree of nonextensivity of the system. In Eq. (5), $k_{B}$ denotes Boltzmann's constant, $W$ is the total number of microscopic states, and $p_{i}$ represents a set of probabilities. At the limit $q=1$, we recover the BG entropy.

By using this formalism, Sotolongo-Costa \& Posadas (2004) and Silva et al. (2006) developed a new approach to describe the distribution of earthquakes with magnitude larger than $m$. According to Scherrer et al. (2015) and first demonstrated in Sarlis et al. (2010), the $b_{s}$ is related to entropic index $q$ by the expression

$$
b_{s}=\frac{2(2-q)}{q-1},
$$

where $b_{s}$ is generally defined in the range between 0.8 and 1.2 (see Sarlis et al. (2010) and references therein), and for the vast majority of systems studied so far, the $q$-index is limited to between 1 and 3 (Abe \& Okamoto, 2001).

The Gutenberg-Richter law is an asymptotic relation between the total number of earthquakes $N$ and the magnitude $m$, given by:

$$
\log \left(N_{m}\right)=a+b_{G R} m,
$$

where the $b_{s}$ values are calculated by Sarlis et al. (2010) and the $b_{G R}$ values are calculated using the Gutenberg-Richter law cited above.

As quoted by Scherrer et al. (2015), the values of $b_{G R}$ are calculated using a software package to analyze the seismicity, denoted by Wiemer $(2001)^{2}$. These authors found that the values of $b_{G R}$ differ from the $b_{s}$-index estimated using a nonextensive approach. They also found that the $q$-values correlated with some properties of subduction zones, such as the occurrence of ruptures, seismic/aseismic slip, coupling, and the interaction of asperities (Scherrer et al., 2015).

\section{CATALOG DATA}

Scherrer et al. (2015) have produced a list of four Circum-Pacific subduction zones distributed in a belt along the so-called Ring of Fire (see Fig. 1 from the aforementioned paper). These data were extracted from the National Earthquake Information Center (NEIC) catalog ${ }^{3}$ (Masse \& Needham, 1989). From that sample, we selected 12 areas to apply our analysis, with 142,280 events in the magnitude interval $1<m<9$ during a decade from
2001 to 2010 (see Table 1). A map with the distribution of the Circum-Pacific subduction zones can be seen in Figure 1 from Scherrer et al. (2015). As reported by Scherrer et al. (2015), the NEIC catalog offers magnitude time series with different magnitudes types $\left(M_{w}, M_{b}, M_{s}, M_{l}\right.$, and $\left.M_{d}\right)$ for the same event. In addition, we decide that using this sequence makes no significant impact on the result of the present paper because the differences between magnitudes types are smaller than 1 . As the $M_{s}$ (surface-wave magnitude) is rarely used, we did not consider the bias from this magnitude (Rezapour \& Pearce, 1998).

The data sample used by Scherrer et al. (2015) is distributed in four different subduction zones defined by asperities and broadness of the rupture front. The main structure of the zones is described by the authors in the aforementioned study. The reader is referred to Scherrer et al. (2015) for details regarding the instrumental procedure and classification.

For the present analysis, we considered earthquakes with a magnitude greater than 1 for all the regions, in this case only the effect due to macroearthquakes is analyzed. Scherrer et al. (2015) measured three important parameters: (i) the entropic index $q$, which emerges from the nonextensive statistical mechanics (Tsallis, 1988; Abe \& Okamoto, 2001; Gell-Mann \& Tsallis, 2004); (ii) the $b_{s}$-index extracted from the slope of the nonextensive Gutenberg-Richter law (Sotolongo-Costa \& Posadas, 2004; Silva et al., 2006); and (iii) the classical index $b_{G R}$ from the Gutenberg-Richter law (Gutenberg \& Richter, 1944) calculated using ZMAP software. In the next section, we compare the Gutenberg-Richter indexes with the Hurst exponent $H^{4}$.

\section{RESULTS AND DISCUSSIONS}

As shown in the top panels in Figures from 1 to 4 , the $R / S$ method was used to estimate the values of the Hurst exponent for a data set of four Circum-Pacific subduction zones. As a result, the values of $H$ were calculated using the slope of the $\log -\log$ plot of $R / S$ versus $n$, over the entire range of $n$ and are summarized in Table 1 .

In the right bottom panels in Figures 1 to 4 , there is a spectrum of the $H$ exponent, which was calculated using a kernel density estimation. As the Kernel density is based on the smoothing functions, we used it to identify the profile of the distribution of the $\mathrm{H}$-index, and therefore to estimate the width of the $H$ distribution. In certain cases, the width of the distribution of $H$ is quite narrow, and therefore the data sample

\footnotetext{
${ }^{2}$ http://www.seismo.ethz.ch/en/research-and-teaching/products-software/software/ZMAP/

3 https://www.usgs.gov/natural-hazards/earthquake-hazards/data-tools

${ }^{4}$ The values of $H$-index were calculated using MATLAB code indicated by the link: https://www.mathworks.com/matlabcentral/fileexchange/39069-hurst-exponentestimation
} 

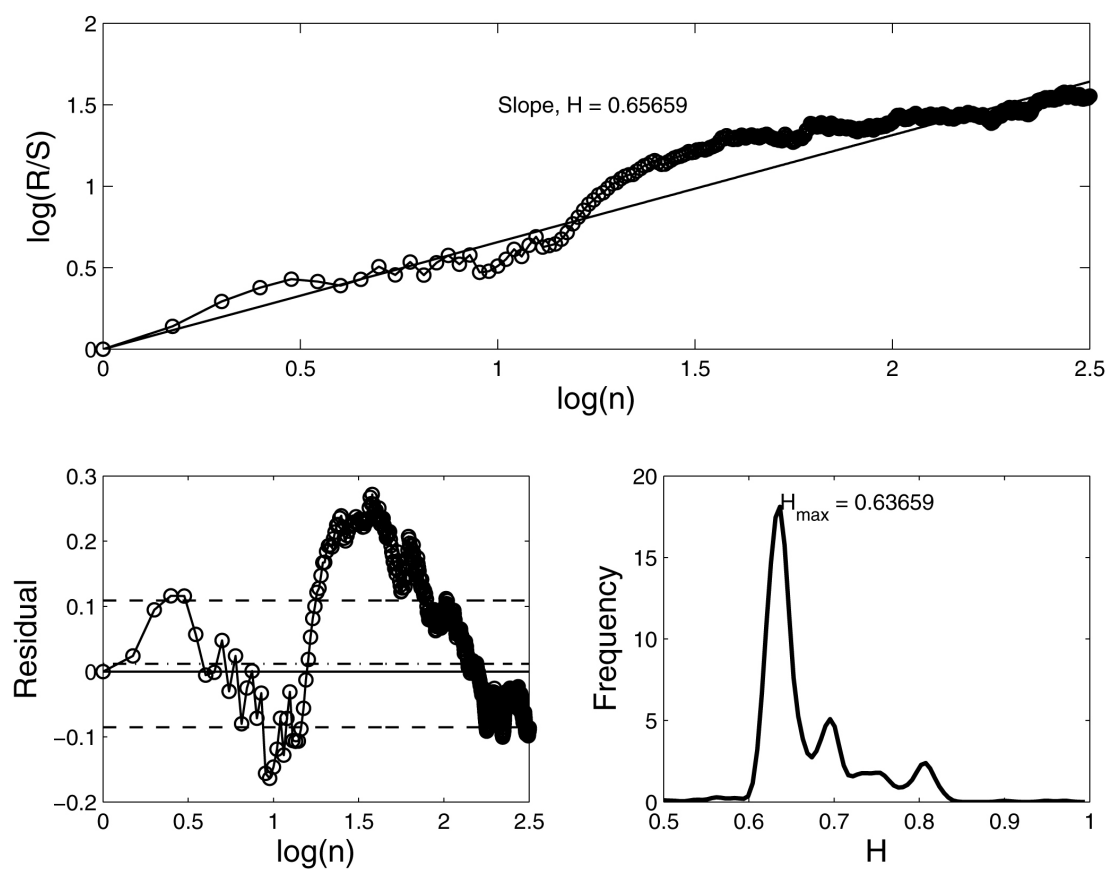

Figure 1 - Top panel: Individual $\log (R / S)$ points as a function of the logarithm of box-size $n$ for Alaska, representing the zone 1. Left bottom panel: Residual extracted from the difference between the $\log (R / S)$ points and the best linear fit in the least-square sense. The solid line represents the perfect agreement, the dashed line denotes the mean value, whereas the dash-dotted lines indicate $1 \sigma$. Right bottom panel: This plot is the Kernel adjustment of $H$ calculated as the derivative of the $R / S$ curve after each iteration $n . H_{\max }$ is the maximum value of the distribution of $H$ which in all cases differs slightly from the value of $H$ identified by a straight line in the top panels.
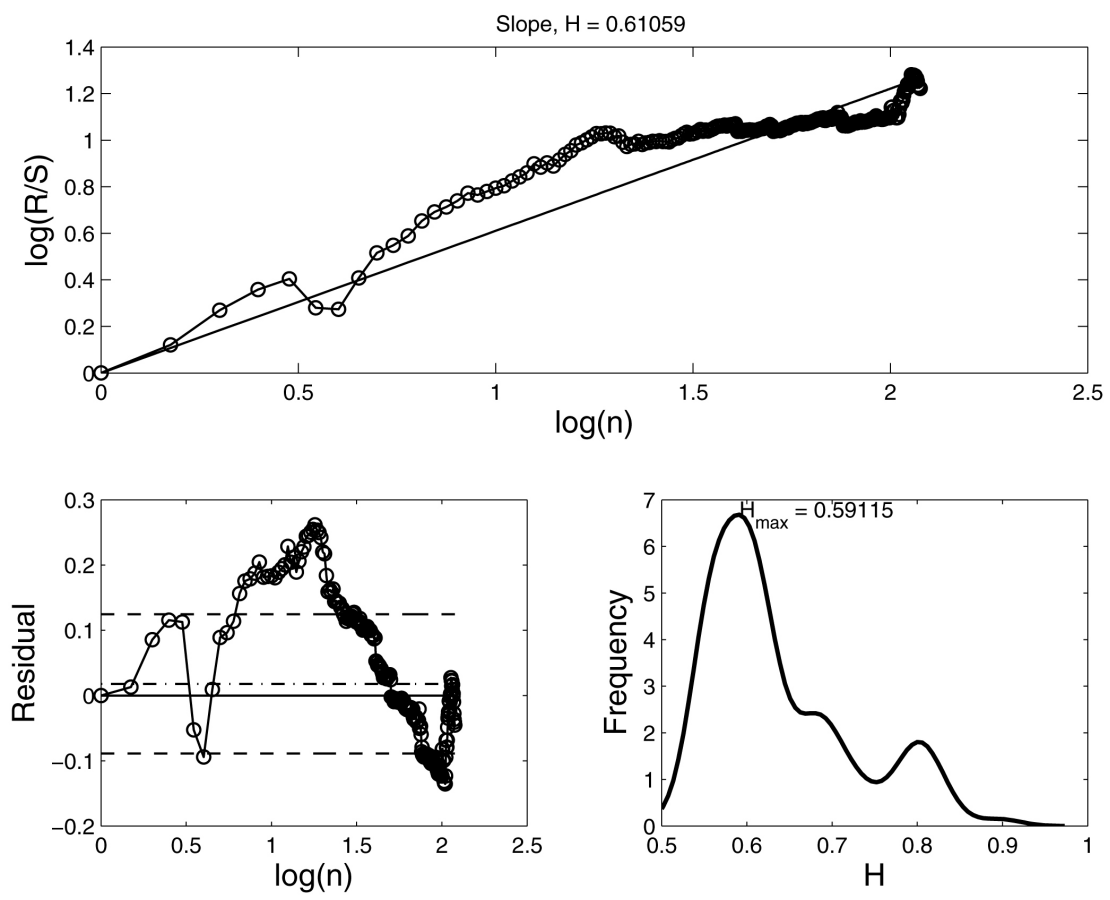

Figure 2 - Fractal analysis for Colombia, representing the zone 2. 

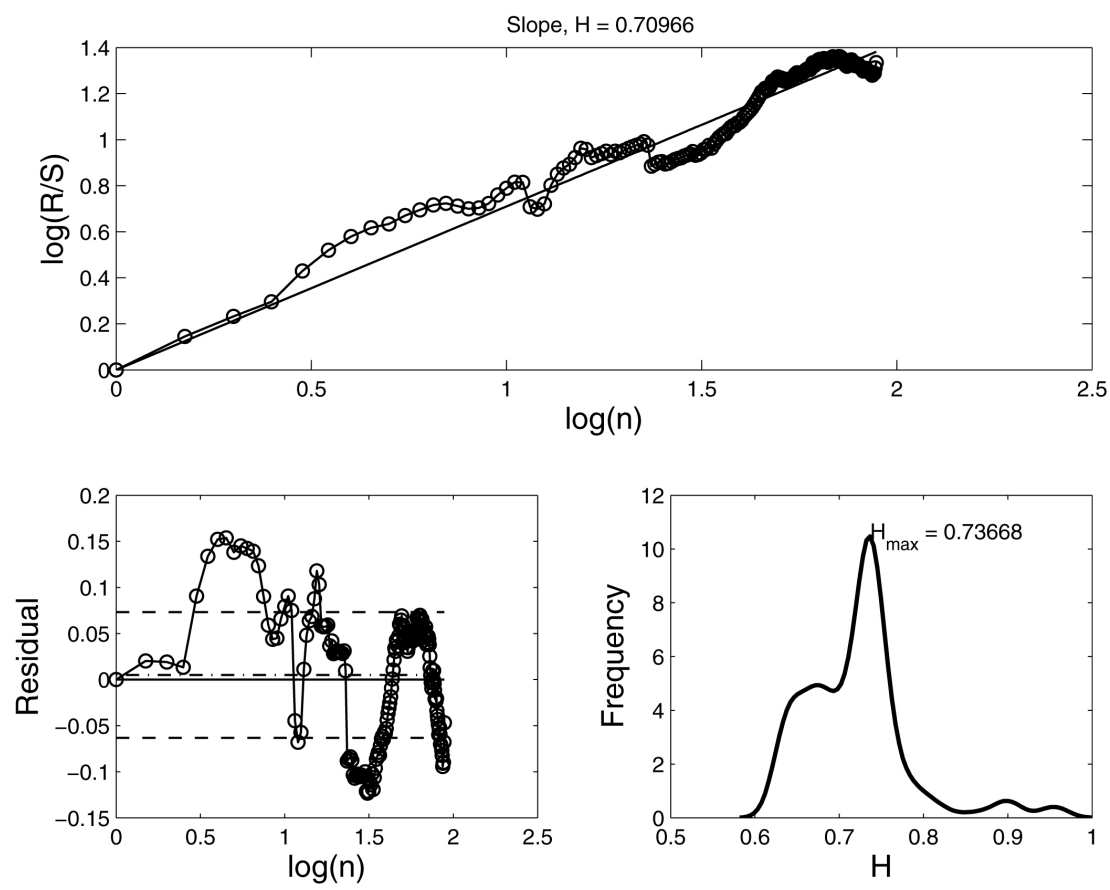

Figure 3 - Fractal analysis for Peru, representing the zone 3.
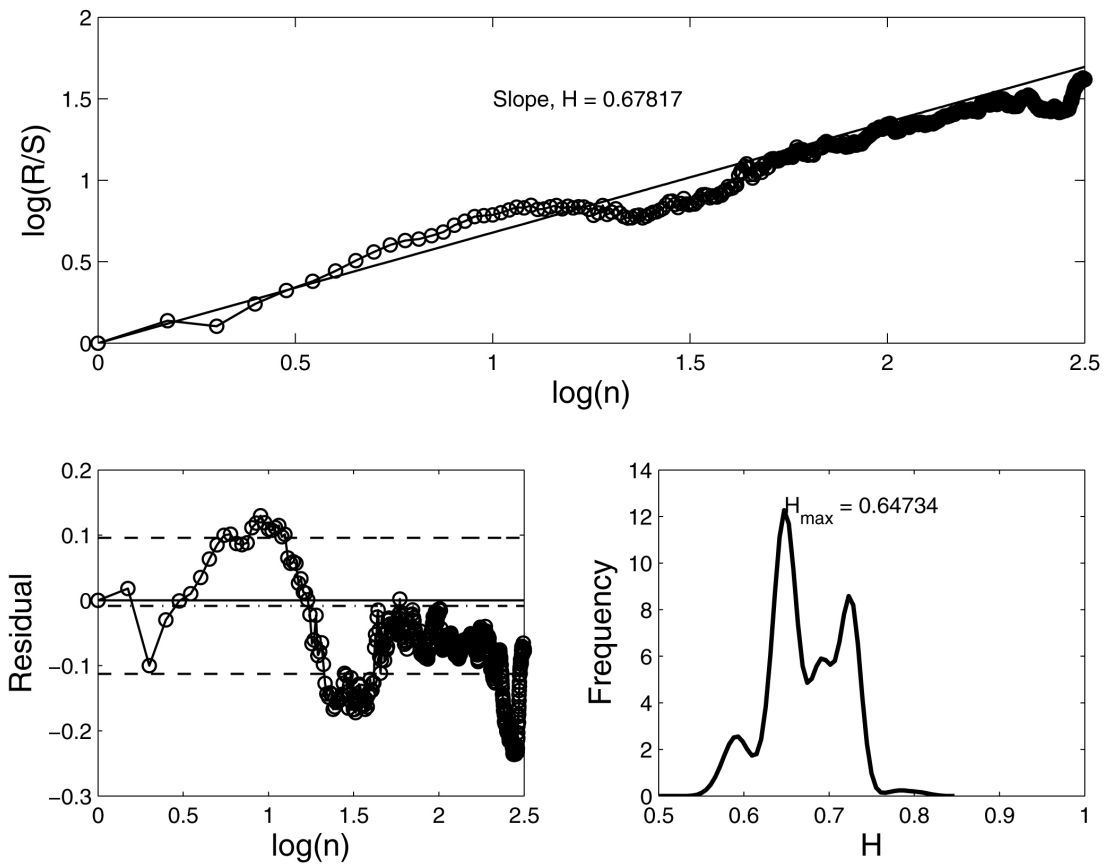

Figure 4 - Fractal analysis for Marianas Islands, representing the zone 4. 
can be considered a fractal. According to the residual panels, such as those shown in Figures 1 to 4 , we found that there is a considerable variation in the value of $H$ along the magnitude series. However, we would have to use multifractal methods for greater precision regarding how narrow the distribution should be in order for the system to be considered a fractal.

We performed a bootstrap resampling method to estimate the $95 \%$ confidence interval in each time series. Firstly, we used a set of 1000 bootstrap replications of the Hurst exponent calculated point to point, i.e., for each ratio of $R(n) / S(n)$ one value of $H$ can be extracted so one time series yields $n-2$ values of $H$. In this case, we have a set of values for $H$, then we performed the bootstrap method. Finally, we ranked the bootstrapped means of $H$, from the lower to the higher value, and took the 25th and the 75th means in the rank as the lower and upper limits of the confidence interval, respectively. The mean Hurst exponents and their symmetrical confidence interval are presented in Table 1.

As seen in Table 1 all the values of these exponents suggest that there is persistence in the subduction zone data, i.e., the values of $H$ are always greater than 0.5 , mostly fluctuating around 0.65 . In this sense, the values of $H>0.5$ might show that earthquakes in the Pacific Ring of Fire are not a Gaussian process, i.e., random variables are not normally distributed. On the contrary, there is a long-term memory associated with the fluctuation dynamics (Li \& Chen, 2001).

Firstly, we verified that only the $b_{s}$-index provides a reasonable correlation with $H$. This relationship can be observed in Figure 5, where we divided the data points into two regimes with different slopes. We fit the following law to these regimes:

$$
H=\frac{A}{b_{s}}+C .
$$

We found the following values for slopes: $A_{\circ}=0.56 \pm 0.11$ and $A_{\bullet}=1.10 \pm 0.10$. The values for the intercepts were: $C_{\circ}=0.18 \pm 0.12$ and $C_{\bullet}=-0.43 \pm 0.11$. We calculated the anti-correlation using the Spearman $\left(r_{S}\right)$ and Pearson $\left(r_{P}\right)$ correlation coefficients and found that $r_{S}^{\circ}=-0.99, r_{P}^{\circ}=$ $-0.96, r_{S}^{\bullet}=-0.74$, and $r_{P}^{\bullet}=-0.97$ (Press et al., 1988). We observed that $A$. was associated with subduction zones with large magnitudes, whereas $A_{\circ}$ was associated with areas with smaller magnitudes. In particular, the values of $H$ can be associated with the mechanism which controls the level of seismic activity.

\section{Is it possible to determine the index $H$ from $b_{S}$ ?}

Inspired by the works of Borland (2002), Sarlis et al. (2010) and Scherrer et al. (2015), and by the empirical relation found in the previous section, we investigated a possible theoretical correlation between the indexes $H$ and $b_{s}$, based on the entropic index $q$.

Borland (2002) introduced a relation between the $q$-index and the exponent $H$ :

$$
H=\frac{1}{3-q}
$$

The above relationship is obtained by using the Langevin equation as a function of the entropic index $q$ (Borland, 2002). This relationship is only valid for $-\infty<q<2$ because the range of the $H$-index is defined between zero and unity.

The Eqs. (6) and (9) suggest that it is possible to determine a relation between $H$ and $b_{s}$. Substituting $q$ from (6) into (9), we have

$$
H=0.5+\frac{0.5}{1+b_{s}}
$$

which indicates an anti-correlation between the indexes.

As we can observe in Figure 5, the above equation is not in agreement with the values found for the parameters $A$ and $C$, which were extracted from an empirical relationship (8). Instead, Eq. (10) can be used to estimate the lower and upper values of $H$. Based on the expected values of $b_{s}$ mentioned in Nonextensive framework, the values of $H$ are limited to between 0.72 and 0.78 . However, these theoretical values of $H$ are an overestimation when compared with the values shown in Table1, indicating that Eq. (10) cannot be used to estimate the values of $H$.

\section{CONCLUSIONS}

We aimed to answer the two questions previously mentioned in the introduction. Firstly, is there a correlation between the $H$-value and the Circum-Pacific subduction zones? Secondly, is there any connection between the fractal parameter and the asperity model from Lay \& Kanamori (2013)? Our study indicates the possible geophysical meaning of the $H$-index in relation to subduction zones. As seen in Results and Discussions, there are two different behaviors for the $H$-value as a function of the parameter $b_{s}$. The explication for these two domains of the value of $H$ may be the lack of large earthquakes during this time period (2001-2010) in the Peru- and Marianas-type zones. In fact, zones 3 and 4 present large amounts of aseismic slip, where there is an inhibition of large rupture development, generating complicated ruptures and foreshock-aftershock activity.

We used the Hurst analysis to investigate the behavior of twelve magnitude catalogs along the Pacific Ring of Fire. From that analysis, we found that there is a relationship between the 
Table 1 - Identifier number of subduction zones (SZ) and $b_{s}$ (see Scherrer et al., 2015), and $H$ and $\sigma_{\text {Kernel }}^{H}$ estimated by our analysis. Symbols o and $\bullet$ are used to differentiate the subsamples shown in Figure 5.

\begin{tabular}{lccc}
\hline Area & $S Z$ & $b_{S} \pm \sigma$ & $H \pm \sigma$ \\
\hline Alaska • & 1 & $1.005 \pm 0.019$ & $0.657 \pm 0.017$ \\
Aleutians Islands• & $1-2$ & $0.965 \pm 0.029$ & $0.709 \pm 0.002$ \\
Central America o & $2-3$ & $1.154 \pm 0.013$ & $0.655 \pm 0.008$ \\
Central Chile • & 3 & $1.054 \pm 0.023$ & $0.600 \pm 0.011$ \\
Colombia • & 2 & $1.054 \pm 0.015$ & $0.611 \pm 0.031$ \\
Kuriles Islands• & 3 & $1.050 \pm 0.042$ & $0.602 \pm 0.016$ \\
Marianas Islandso & 4 & $1.150 \pm 0.015$ & $0.678 \pm 0.009$ \\
New Hebrides • & $2-3$ & $1.010 \pm 0.008$ & $0.665 \pm 0.030$ \\
Peru o & 3 & $1.049 \pm 0.015$ & $0.710 \pm 0.020$ \\
Solomon Islands • & 2 & $1.074 \pm 0.019$ & $0.605 \pm 0.013$ \\
Tonga \& Kermadec $\circ$ & 4 & $1.210 \pm 0.010$ & $0.636 \pm 0.006$ \\
\hline
\end{tabular}

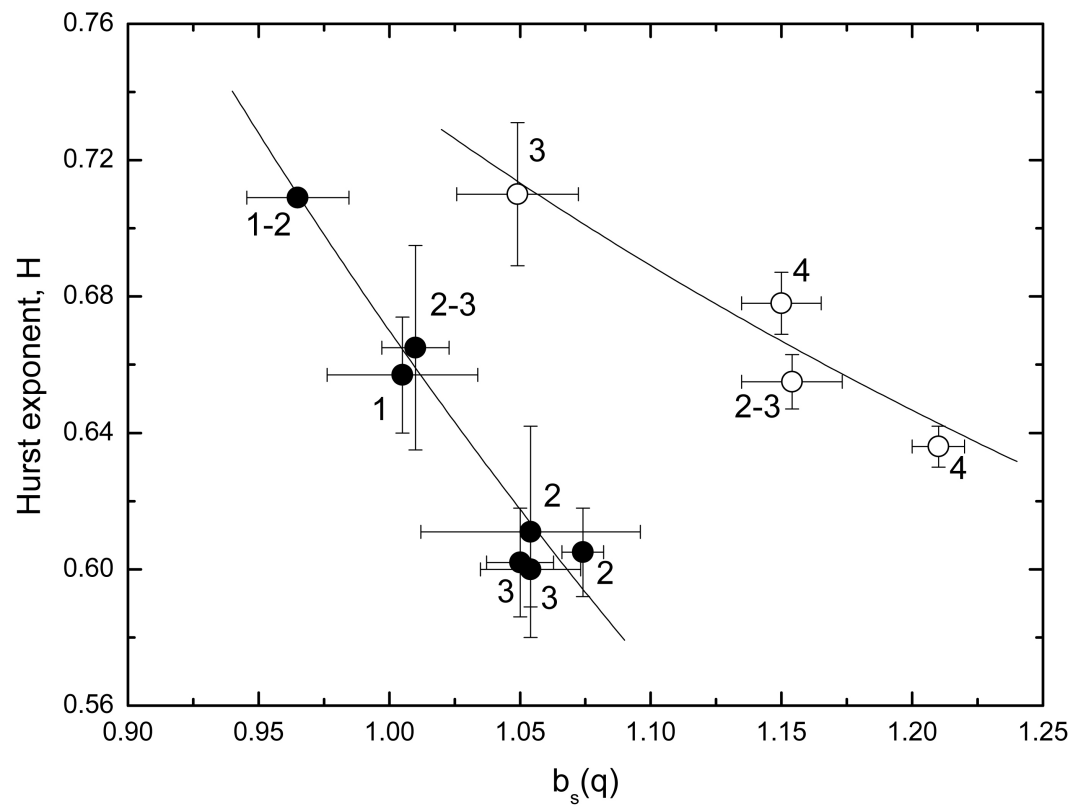

Figure $\mathbf{5}$ - Values of the $b_{s}$-index extracted from the slope of the modified Gutenberg-Richter law as a function of $H$-index. Numbers indicate the category of each subduction zone. Solid and open circles are used to distinguish the two different domains. The numbers 1 to 4 denote 4 subduction zones indicated by Table 1. 
Hurst exponent $H$ and the modified Gutenberg-Richter index $b_{s}$, as illustrated by Figure 5 .

Our results reveal a strong long-range persistence in the magnitudes series studied, indicated by the values of $H$ greater than 0.5 . The long-range persistence shows that the coupling between random variables in magnitude series at different times is stronger than the short-range one. In other words, the persistent behavior in the time series characterized by calculating the Hurst exponent reveals the existence of the persistence of long-range memory. In addition, there is a statistical correlation between the subduction zones, as shown in Figure 5.

The existence of an empirical correlation between the Hurst exponent and the $b_{s}$-index opens up the possibility of proposing a better model for seismic hazard and risk assessments with high memory, which these systems present. In addition, we can conclude that the dynamics associated with fragment-asperity interactions can be emphasized as a self-affine fractal phenomenon.

\section{REFERENCES}

ABE S \& OKAMOTO Y. 2001. Nonextensive statistical mechanics and its applications. Volume 560. Berlin Springer Verlag. 278 pp.

ALESSIO E, CARBONE A, CASTELLI G \& FRAPPIETRO V. 2002. Second-order moving average and scaling of stochastic time series. European Physical Journal B, 27(2): 197-200.

BORLAND L. 2002. Option Pricing Formulas Based on a Non-Gaussian Stock Price Model. Physical Review Letters, 89(9): 098701.

DE FREITAS DB, FRANÇA G, SCHERRER T, VILAR C \& SILVA R. 2013. Nonextensive triplet in a geological faults system. EPL (Europhysics Letters), $102(3): 39001$.

DE FREITAS DB, LEÃO I, FERREIRA LOPES C, PAZ-CHINCHON F, MARTINS BC, ALVES S, DE MEDEIROS J \& CATELAN M. 2013. New Suns in the Cosmos? ApJ, 773(2): L18.

DE FREITAS DB, NEPOMUCENO MMF, DE SOUZA MG, LEÃO IC, CHAGAS ML, COSTA A, MARTINS BLC \& DE MEDEIROS JR. 2017. New Suns in the Cosmos. IV. The Multifractal Nature of Stellar Magnetic Activity in Kepler Cool Stars. ApJ (Astrophysical Journal), 843(2): 103. $13 \mathrm{pp}$.

GELL-MANN M \& TSALLIS C. 2004. Nonextensive entropy: interdisciplinary applications. Oxford University Press on Demand. 422 pp.

GUTENBERG B \& RICHTER CF. 1944. Frequency of earthquakes in California. Bull. Seismol. Soc. Am., 34(4): 185-188.

HURST HE. 1951. Long-term storage capacity of reservoirs. Trans. Amer. Soc. Civil Eng., 116: 770-799.
KAWAMURA H, HATANO T, KATO N, BISWAS S \& CHAKRABARTI BK. 2012. Statistical physics of fracture, friction, and earthquakes. Reviews of Modern Physics, 84(2): 839-884.

LAY T \& KANAMORI H. 2013. An asperity model of large earthquake sequences. American Geophysical Union, p. 579-592.

LI J \& CHEN Y. 2001. Rescaled range $(R / S)$ analysis on seismic activity parameters. Acta Seismologica Sinica, 14(2): 148-155.

MANDELBROT BB. 1985. Self-affine fractals and fractal dimension. Physica Scripta, 32(4): 257.

MANDELBROT BB \& WALLIS JR. 1969a. Robustness of the rescaled range $R / S$ in the measurement of noncyclic long run statistical dependence. Water Resources Research, 5(5): 967-988.

MANDELBROT BB \& WALLIS JR. 1969b. Some Long-Run Properties of Geophysical Records. Water Resources Research, 5(2): 321-340.

MASSE R \& NEEDHAM R. 1989. NEIC - The National Earthquake Information Center. Earthquakes \& Volcanoes (USGS), 21(1): 4-44.

OMORI F. 1894. On the after-shocks of earthquakes. Volume 7. J. Coll. Sci. Imp. Univ. Tokyo.

PEITGEN HO, JÜRGENS H \& SAUPE D. 2004. Classical Fractals and Self-Similarity. In: PEITGEN HO, JÜRGENS H \& SAUPE D (Eds.). Chaos and Fractals: New Frontiers of Science. Springer, New York, NY. p. 61-128.

PENG CK, BULDYREV SV, GOLDBERGER AL, HAVLIN S, SCIORTINO F, SIMONS M \& STANLEY HE. 1992. Long-range correlations in nucleotide sequences. Nature, 356(6365): 168-170.

PRESS WH, FLANNERY BP, TEUKOLSKY SA \& VETTERLING WT. 1988. Numerical Recipes in C: The Art of Scientific Computing. Cambridge University Press. $735 \mathrm{pp}$.

REZAPOUR M \& PEARCE RG. 1998. Bias in surface-wave magnitude Ms due to inadequate distance corrections. Bull. Seismol. Soc. Am., 88(1): 43-61.

SARLIS N, SKORDAS E \& VAROTSOS P. 2010. Nonextensivity and natural time: The case of seismicity. Physical Review E, 82(2): 021110.

SCHERRER TM, FRANÇA GS, SILVA R, DE FREITAS DB \& VILAR CS. 2015. Nonextensivity at the Circum-Pacific subduction zones - Preliminary studies. Physica A: Statistical Mechanics and its Applications, 426: 63-71.

SEURONT L. 2009. Fractals and Multifractals in Ecology and Aquatic Science. Taylor \& Francis. Boca Raton, FL. 364 pp.

SILVAR, FRANÇA G, VILAR C \& ALCANIZ J. 2006. Nonextensive models for earthquakes. Phys. Rev. E, 73(2): 026102.

SOTOLONGO-COSTA 0 \& POSADAS A. 2004. Fragment-asperity interaction model for earthquakes. Physical Review Letters, 92(4): 048501. 
SUYAL V, PRASAD A \& SINGH HP. 2009. Nonlinear time series analysis of sunspot data. Solar Physics, 260(2): 441-449.

TANG L, LV H, YANG F \& YU L. 2015. Complexity testing techniques for time series data: A comprehensive literature review. Chaos, Solitons and Fractals, 81: 117-135.

TELESCA L. 2016. Fractal Methods in the Investigation of the Time Dynamics of Fires: An Overview. In: DIMRI V (Ed.). Fractal Solutions for Understanding Complex Systems in Earth Sciences. Springer Earth System Sciences. Springer, Cham. p. 117-152.

Recebido em 27 de setembro de 2018 / Aceito em 15 de novembro de 2019

Received on September 27, 2018 / Accepted on November 15, 2019
TELESCA L, COLANGELO G \& LAPENNA V. 2005. Multifractal variability in geoelectrical signals and correlations with seismicity: a study case in southern Italy. Natural Hazards and Earth System Sciences, 5(5): 673-677.

TSALLIS C. 1988. Possible generalization of Boltzmann-Gibbs statistics. Journal of Statistical Physics, 52(1-2): 479-487.

WIEMER S. 2001. A software package to analyze seismicity: ZMAP. Seismol. Res. Lett., 72(3): 373-382. 